\title{
X-ray lenses fabricated by deep x-ray lithography
}

\author{
Derrick C. Mancini, ${ }^{\mathrm{a}}$ Nicolaie Moldovan, ${ }^{\mathrm{a}}$ Ralu Divan, ${ }^{\mathrm{a}}$ Francesco DeCarlo, ${ }^{\mathrm{b}}$ Judith Yaeger ${ }^{\mathrm{a}}$ \\ Optics Fabrication and Metrology Group, ${ }^{\mathrm{a}}$ Microtechniques Group, \\ Experimental Facilities Division, \\ Advanced Photon Source, Argonne National Laboratory, \\ 9700 S. Cass Avenue, Argonne IL 60439
}

\begin{abstract}
Refractive X-ray lenses have been fabricated using deep x-ray lithography. Lenses were produced directly in 1- to 6-mm-thick sheets of polymethylmethacrylate (PMMA) with as many as 100 cylindrical lenses along the optical axis. The fabrication process consists of exposing the PMMA sheets to highenergy synchrotron radiation through a mask of 50-micron-thick gold on silicon and subsequent development in ketone. The lenses are suitable for use in synchrotron radiation from a bending magnet at the Advanced Photon Source in the energy range of 8-16 keV. Results of measurements of focus quality, flux density gain, and scatter are presented and discussed with regard to the quality of lens material and fabrication method. Means for improving the performance of the lenses is discussed.
\end{abstract}

Keywords: $x$-ray lens, x-ray lithography, LIGA, x-ray focusing

\section{INTRODUCTION}

Deep x-ray lithography (DXRL) provides the means for precision microfabrication of optical components with high aspect ratio. ${ }^{1}$ The realization of compound refractive lenses for x-rays ${ }^{2}$ was soon followed by the suggestion that DXRL could be used for the fabrication of refractive X-ray lenses. ${ }^{3,4}$ The fabrication of X-ray lenses using DXRL has been reported by Kupka, et al., ${ }^{5}$ with an analysis of the contribution of the x-ray optical properties of various materials and lens design on the theoretical performance of such lenses. Both cylindrical and parabolic designs were realized in resist material less than $1 \mathrm{~mm}$ thick, although no details on measured performance were reported. The Advanced Photon Source (APS) provides the higher energy x-rays required for DXRL for thicknesses greater than millimeter up to a centimeter. First results for the fabrication of x-ray lenses at least $1 \mathrm{~mm}$ thick at the APS were limited to cylindrical lenses with circular figure. ${ }^{6}$ The results were encouraging enough, however, for the creation of new mask sets and fabrication of lenses of improved design. Moreover, since the many refracting surfaces of the compound lens each contribute to the focusing, their defects add in such as way as to be a very sensitive test of the quality of DXRL in its ability to accurately reproduce planar geometries with minimum defects. 


\section{LENS DESIGN}

The first lenses fabricated at the APS using DXRL were cylindrical lenses with circular figure, $1 \mathrm{~mm}$ radius of curvature, aperture of $1 \mathrm{~mm}$, septa width of $\sim 40 \mu \mathrm{m}$, and with 85 biconvex lenses on a common optical axis. ${ }^{6}$ There was no attempt to optimize the figure, with the primary purpose being to test the ability to fabricate the lenses in free-standing sheets of polymethylmethacrylate (PMMA) and demonstrate their ability to provide focusing and gain. The next set of lenses were designed to include optimization of the compound lens design for best figure, efficiency, and ease of use.

We performed simple calculations to compare the figure of rigorously stigmatic lenses, parabolic lenses, and circular lenses and determine if the variation between the figures is significant compared to the accuracy we expect to achieve with DXRL. For a totally stigmatic lens array, we considered forming an axially stigmatic image of a point source (an idealized synchrotron radiation source) placed 50 meters ahead of the first lens. The lens array is composed of stigmatic biconvex lenses, each using as an object the point-source image produced by the previous lens in the row. As long as we respect this rule in designing the individual lenses, adding new lenses in the row preserves the stigmatism of the system. We considered lenses of the same radius of curvature $\mathrm{R}=1 \mathrm{~mm}$, placed face-to-face pairwise to form a 300- $\square \mathrm{m}$-thick biconvex lens, with gaps of $20 \square \mathrm{m}$ between the lenses. The calculation was made for 100 such lenses.

We compared this with a compound lens formed by 100 biconvex parabolic lenses, given by the equation $\mathrm{z}=\mathrm{y}^{2} / 2 \mathrm{R}$, and a compound lenses formed by 100 cylindrical lenses, of radius $\mathrm{R}$. A parabolic lens would correspond to a stigmatic lens if the point source were infinitely far away. However, a row of parabolic lenses is no longer a stigmatic system, since the object of the second lens is no longer at infinity and so forth.

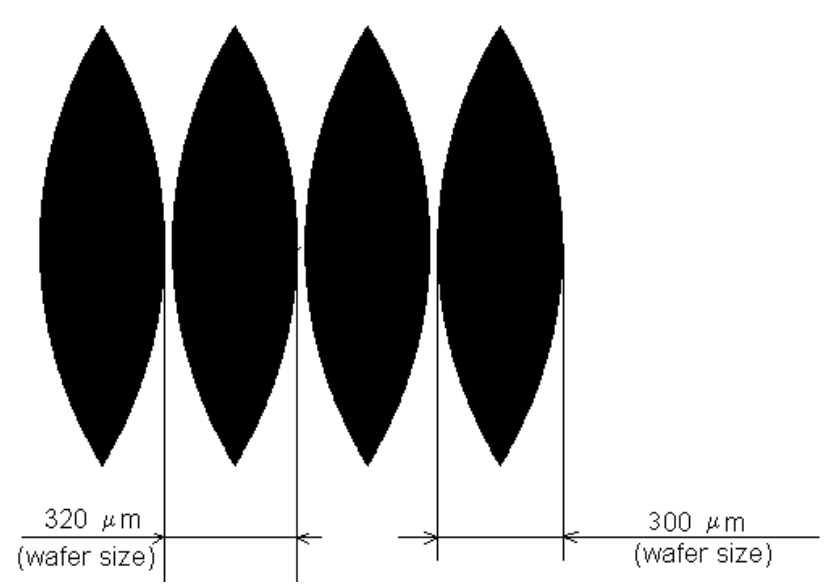

Figure 1. Design of the biconvex lens elements in the compound lenses.

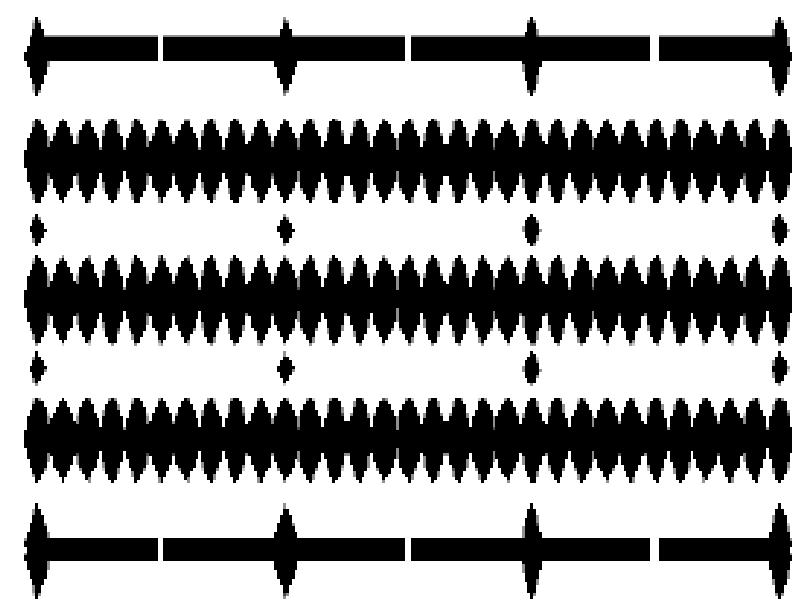

Figure 2. Layout of the compound lenses showing separation features.

When the calculated figure of the stigmatically corrected lenses is compared with that of the parabolic lenses, the variation ranges between $10 \mathrm{~nm}$ to $50 \mathrm{~nm}$, which is well below the accuracy that can be obtained by DXRL. The difference between the parabolic and the circular lenses, however, reaches a 
maximum of $21 \square \mathrm{m}$ toward the outer part of the $1 \mathrm{~mm}$ aperture. This difference is significant for DXRL, which can obtain one micron placement accuracy. Hence, we chose to use parabolic biconvex planar lenses in the optimized design. Moreover, to reduce the absorption losses, the septa between lenses were reduced in half to $20 \mu \mathrm{m}$ (figure 1).

For the purpose of ease of use, distribution, and testing, the final design incorporates arrays of three compound lenses each containing 100 biconvex lenses. Each group of three compound lenses includes two compound lenses composed of planar parabolic lenses and one compound lens composed of circular lenses to allow easy comparison of the performance of parabolic to circular lenses. Every group of three compound lenses is separated from adjacent groups of three by a parallel row of $\sim 3$-mm-long rectangular holes to allow easy separation of groups of lenses by cutting or snapping them apart (figure 2). In addition, small diamond-shaped holes are spaced between rows every tenth lens, to facilitate removal of lenses along the optical axis in groups of ten, allowing easy adjustment of the number of lens elements and thus the focusing power.

\section{MASK FABRICATION}

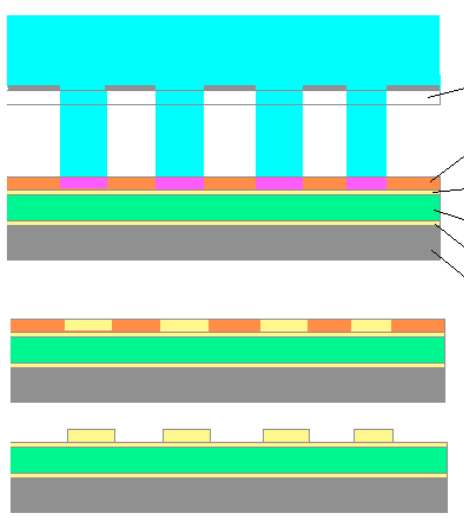

\section{EXPOSURE}

OPTICAL MASK

PHOTORESIST $(5 \mu \mathrm{m})$

Au PLATING BASE

PMMA $(80-100 \mu \mathrm{m})$

PHOTORESIST $(80 \mu \mathrm{m})$

Au PLATING BASE

Si SUBSTRATE $(250 \mu \mathrm{m})$

DEVELOPING \&

ELECTROFORMING

PHOTORESIST

REMOVAL
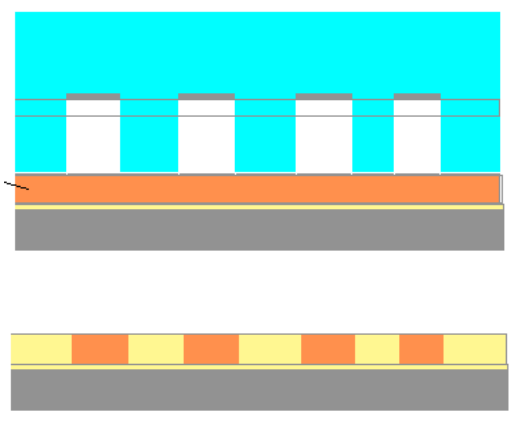
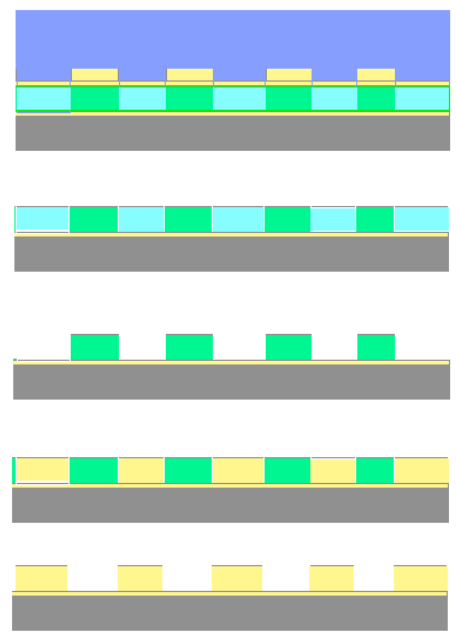

Au REMOVAL

PMMA

DEVELOPING (GG)

SOFT X-RAY

EXPOSURE

Au

ELECTROFORMING

PMMA REMOVAL
Figure 3. Processing description for the fabrication of hard x-ray masks used for DXRL. UV optical lithography is used to form a conformal soft x-ray mask (left) or to directly produced a gold absorber in a thick novolac resist (right). Soft $\mathrm{x}$-ray lithography is used to transfer the pattern into thick PMMA from the conformal soft x-ray mask (lower left). 
The masks used for DXRL were fabricated by two different methods starting from a chrome-on-quartz optical mask with a pattern generated by e-beam lithography. The optical mask was used to transfer the pattern by proximity contact UV-optical lithography into a positive-tone novolac-DNQ resist (Shipley Microposit). In the first method (figure 3, right), the final x-ray mask was produced directly by electroforming gold into the patterned resist spun to thicknesses of 45-80 $\mu \mathrm{m}$ onto a $250-\mu \mathrm{m}$-thick silicon wafer substrate. The final gold thickness was 35-50 $\mu \mathrm{m}$ after stripping remaining photoresist. In the second method (figure 3, left), a soft x-ray mask was produced directly on a thick layer of PMMA by sputter depositing a thin layer of gold onto the PMMA. A thinner layer of novolac resist $(5-8 \mu \mathrm{m})$ was spun onto the sputtered gold layer, exposed, and developed. A conformal soft x-ray maks was formed by electroforming 5- $\mu$ m-thick gold into the patterned resist. This pattern was subsequently transferred into the thick PMMA layer by exposure through the conformal mask with soft x-rays. The gold
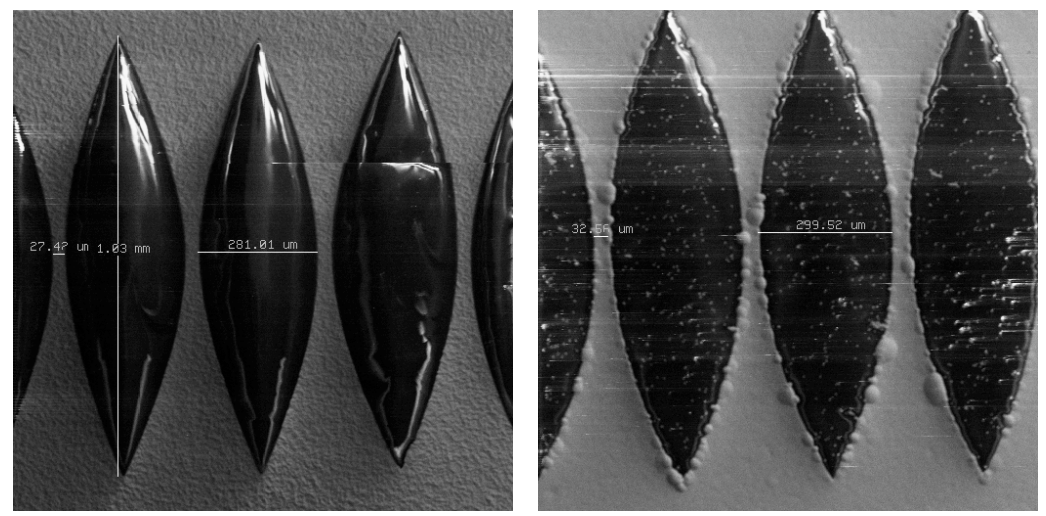

Figure 4. Optical micrographs of hard $\mathrm{x}$-ray masks consisting of $35-50 \mu \mathrm{m}$ gold on $250 \mu \mathrm{m}$ silicon substrate fabricated using (left) soft x-ray lithography in PMMA and (right) UV optical lithography in novolac resist.

conformal mask is removed, the PMMA is developed, and the final hard x-ray mask is formed by electroforming 50- $\mu \mathrm{m}$-thick gold into the patterned PMMA and then stripping the remaining PMMA resist from the $250-\mu \mathrm{m}$-thick silicon wafer substrate.

Both mask fabrication methods produced complete masks with sufficient contrast for DXRL replication. Close examination of the features, however, shows that the masks produced by soft x-ray transfer more accurately replicate the structures, particularly in the narrow region of the septa between lenses (figure 4). Moreover, masks produced directly by UV optical lithography show a greater roughness and number of small defects that could affect performance. ${ }^{7}$ For this reason, a mask fabricated by soft x-ray transfer was used for producing the final parts for testing.

\section{LENS FABRICATION}

Compound x-ray refractive lenses were fabricated in sheets of PMMA using the masks described above. The $\mathrm{x}$-ray exposures were performed at the APS bending magnet beamline 2-BM. ${ }^{8}$ The beam size was $10 \mathrm{~cm} \times 6 \mathrm{~mm}$, and the photon energy was 10-20 keV, after passing through a 1-mm-thick carbon filter and reflecting from a $0.15^{\circ}$ grazing-incidence chromium mirror.

Most of the exposures with the new mask were carried out with 2.5-mm-thick PMMA sheets (Goodfellow, CQ-grade), while earlier exposures used the same material with thicknesses of 1, 3, and 6 $\mathrm{mm}$. Typical exposures were carried out at $100 \mathrm{~mA}$ beam current, providing a total estimated dose of 8,000-12,000 joules/cc in 8-12 hours. After exposure, the sheets of PMMA were developed and rinsed at 

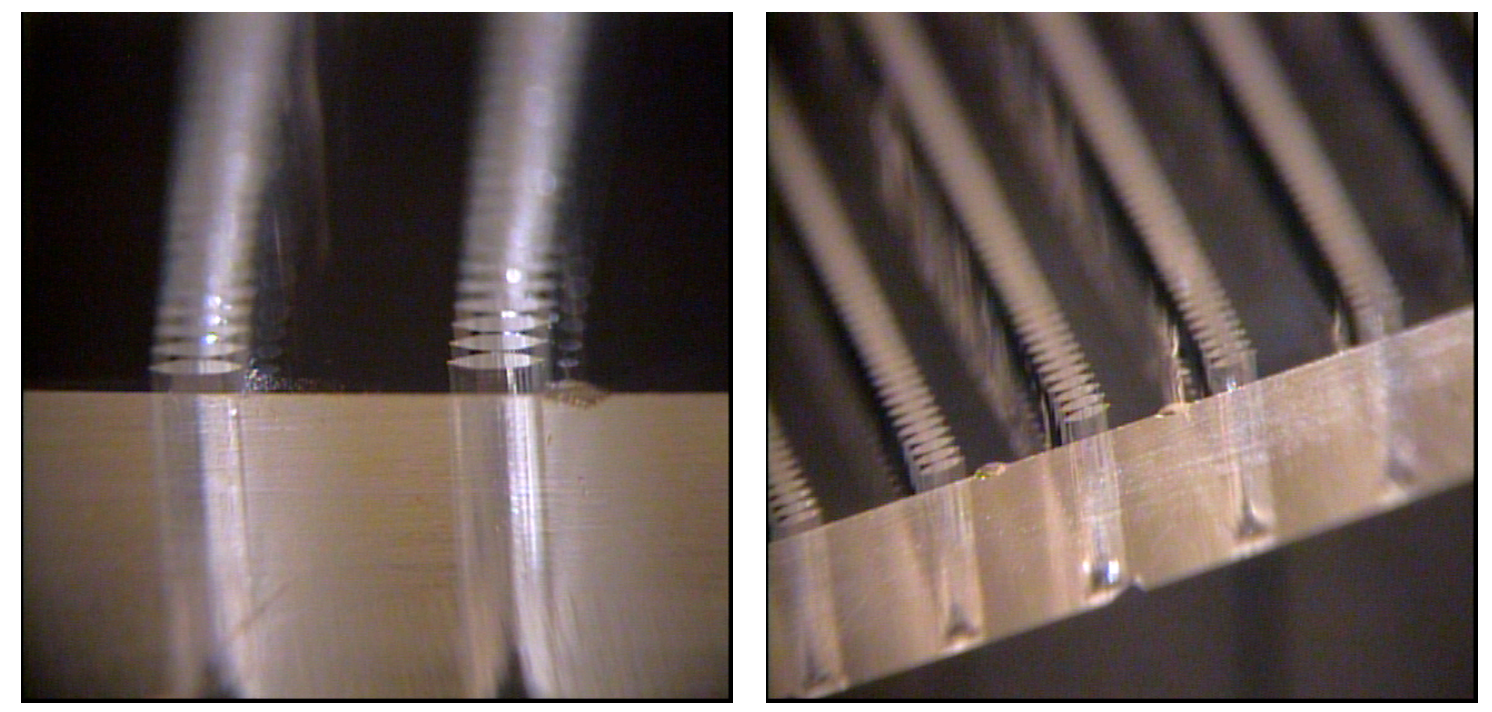

Figure 5. Compound cylindrical $x$-ray lenses fabricated in 3-mm-thick (left) and 6-mm-thick (right) PMMA sheets using deep x-ray lithography.

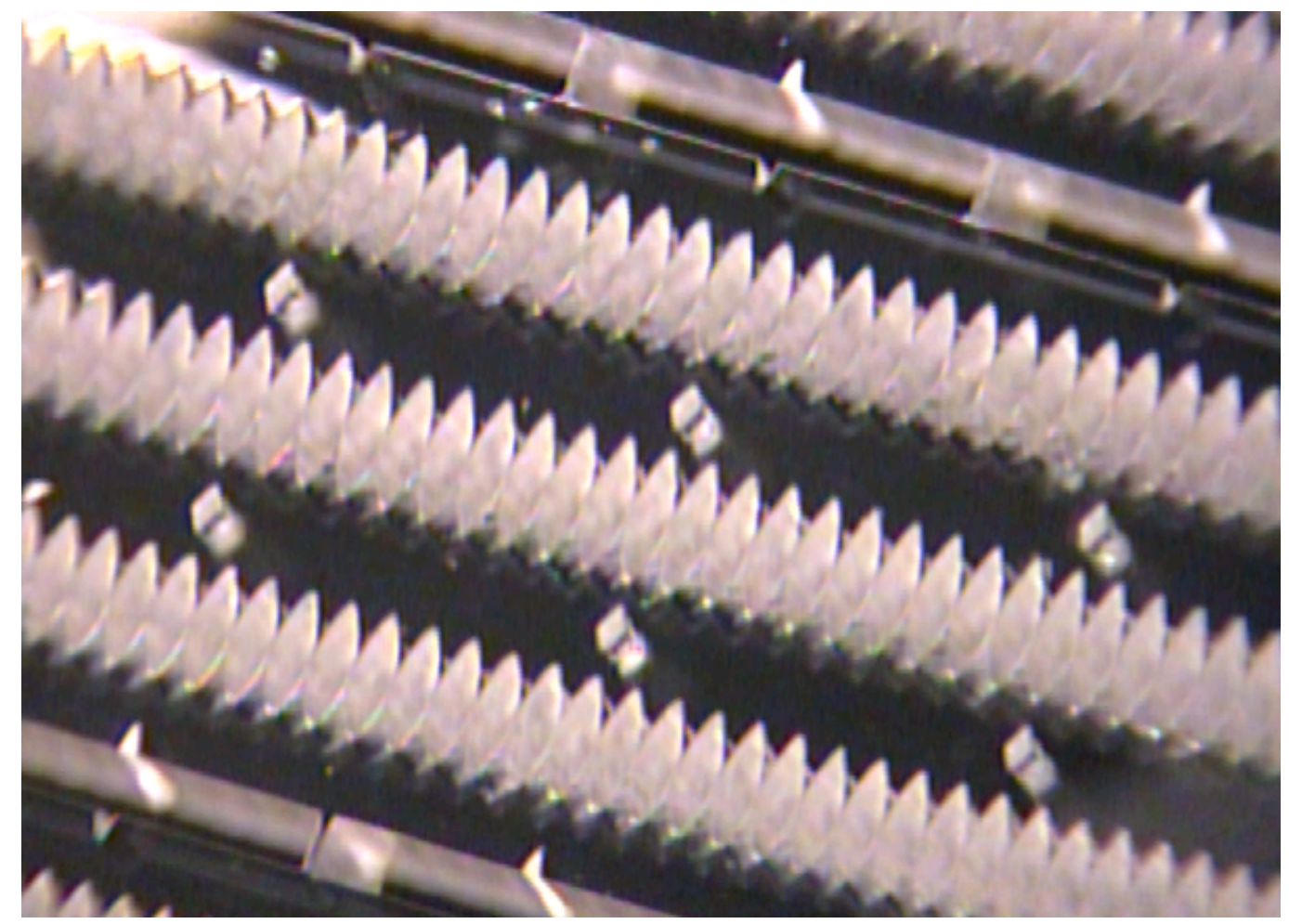

Figure 6. Compound planar parabolic x-ray lenses of a new design fabricated by DXRL in 2.5-mm-thick PMMA, with apertures for separation of lenses. 
$25^{\circ} \mathrm{C}$ in the GG development system, ${ }^{9,10}$ until the lens structures were developed through. The developed sheets were dried after a final deionized water rinse.

Examination of the structures with optical microscopy indicated that the lens structures were accurately reproduced in PMMA sheets for all thicknesses ranging from $1 \mathrm{~mm}$ through $6 \mathrm{~mm}$. The structures exhibited vertical wall profiles, with variations in wall placement of less than $1 \mu \mathrm{m} / \mathrm{mm}$. The septa were very well reproduced in the earlier structures with $40 \mu \mathrm{m}$ width, even for 6-mm-thick sheets (figure 5). For the thinner $25 \mu \mathrm{m}$ septa used for the newer design, however, there did appear to be some variations in the thickness or accuracy of the septa walls on the order of 1-2 $\mu \mathrm{m}$. Nevertheless, the new design was successfully fabricated in larger areas (figure 6) and could be separated by cutting or snapping apart groups of lenses from the sheet.

\section{LENS CHARACTERIZATION}

Various of the compound $\mathrm{x}$-ray refractive lenses fabricated by the methods described above were tested using monochromatic synchrotron radiation from a bending magnet beamline $2 \mathrm{BM}$ at the APS. ${ }^{8}$ The monochromatic radiation is provided by a Kohzu double crystal monochromator using $\mathrm{Si}<111>$ Bragg reflection and a $\mathrm{Cr}$ mirror to reject the third harmonic. The beam size is reduced by aperturing to illuminate a single compound lens using horizontal and vertical slit jaws. The beam is imaged by converting the x-rays to visible light with a thin scintillator screen and using a microscope objective to produce a magnified image of the scintillator on a cooled CCD camera (figure 7). This allows digitization of up to a $1 \mathrm{~K} \times 1 \mathrm{~K}$ pixels with resolution of $1-5 \mu \mathrm{m} / \mathrm{pixel}$, depending on the power of the microscope objective. The lenses are positioned and aligned with an assembly of motorized stages (figure 7). A horizontal $x-y$ translation stage is mounted on a motorized goniometer with 2-axis rotation about the $\mathrm{x}$ and $\mathrm{y}$ directions. The goniometer is mounted on stages that allow vertical translation and rotation in the horizontal plane. The entire assembly is mounted on a horizontal translation stage for extended motions along the optical axis. With this system, the lenses can be aligned to the optical axis

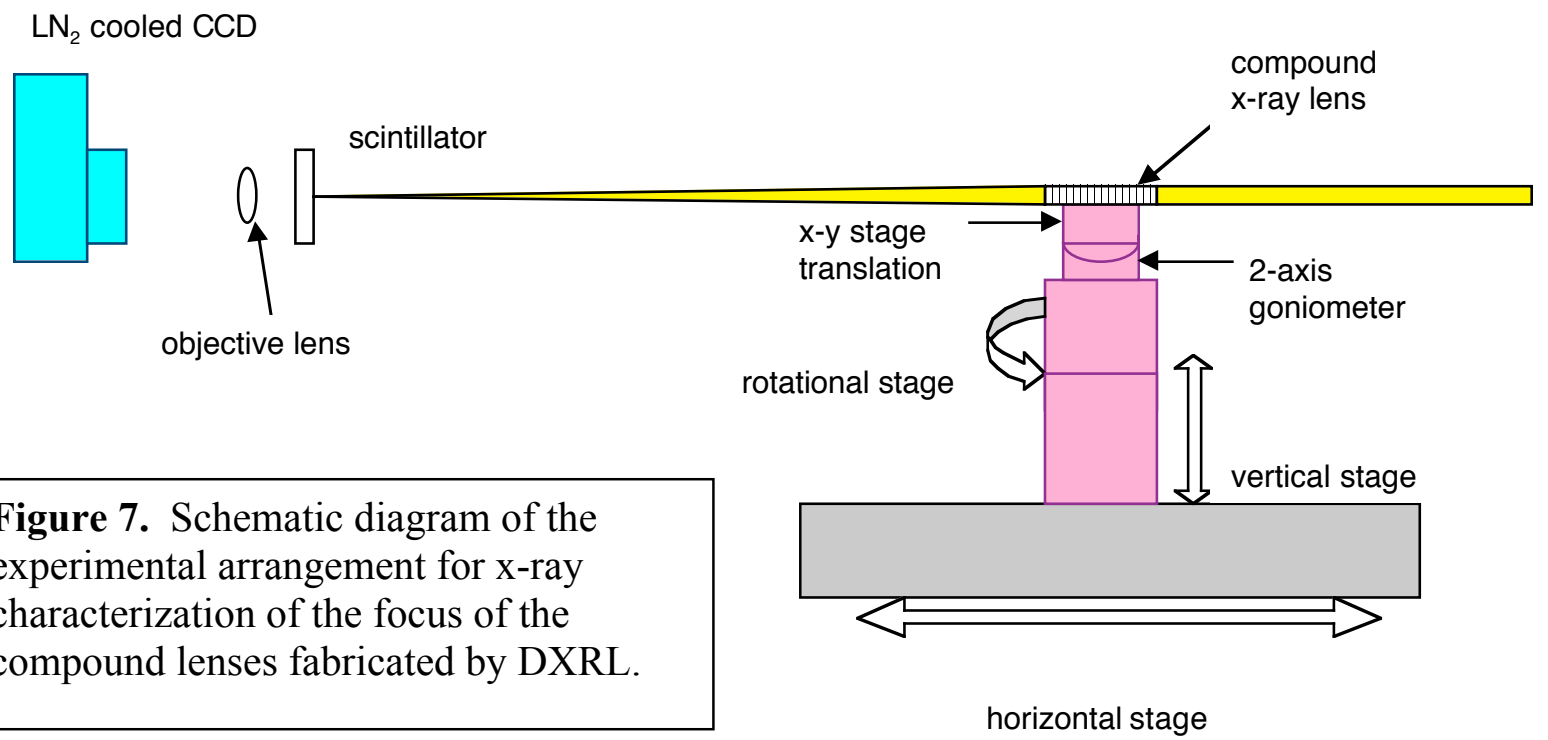


of the x-ray beam. The focal properties can be studied either by scanning the location of the lens along the optical axis at a fixed energy, or by scanning the energy of the monochromator at a fixed location of the lenses.

Gross alignment consists of manual placement of the lens within a couple degrees, and adjustment to increase the apparent brightness of the focused line. During fine alignment, fringes were observed near the top of the lens when slightly misaligned in pitch (figure 8). Accurate alignment of pitch can be achieved by minimizing these fringes. The symmetry of the fringes is very sensitive to misalignments in yaw (horizontal rotation). Hence, very accurate angular alignment could be achieved in this way. Once aligned with the optical axis of the x-ray beam, the focus could be found by translating the lens along the x-ray beam or scanning the energy of the monochromator. Once the optimal focus was found, the azimuthal angle of the lense was adjusted to align the vertical focus of the lens with the pixel orientation of the CCD camera. This allowed statistical averaging of the focus over the aperture of the lens by summing the intensity of the pixels in each vertical column of pixels in the camera (figure 9). The fullwidth-at-half-maximum (FWHM) of the so-measured peak can be used to define the width of the beam, and the narrowest width attained defines the focus. A series of such peaks at different positions when plotted as an $\mathrm{x}-\mathrm{y}$ map of intensity provides a direct visual image of the waist of the focus (figure 10).

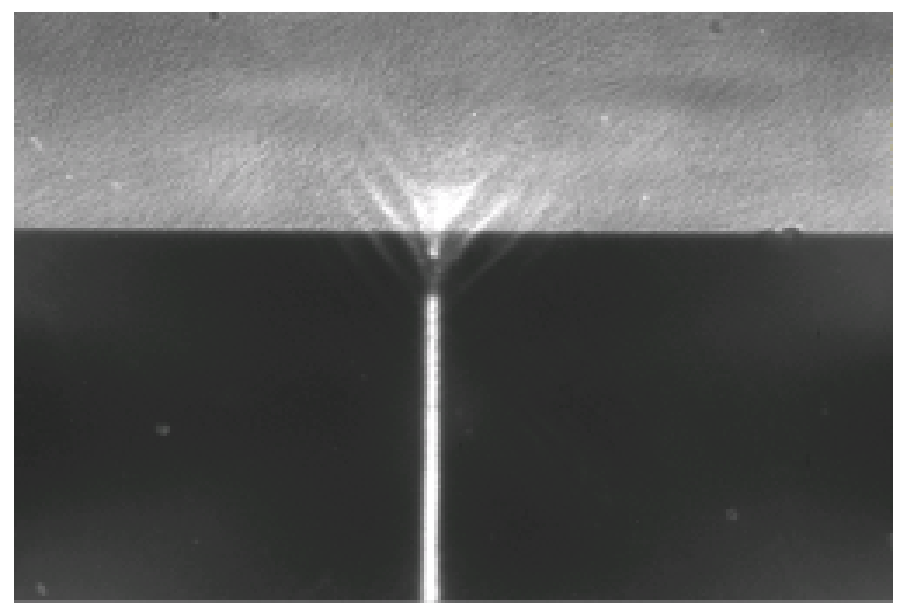

Figure 8. Focus of a compound refractive x-ray lens. Slight misalignment in pitch of the lens produces fringes that appear near the top edge. Small horizontal rotation of the lens shifts the fringes appreciably, so that appearance and shape of the fringes are a sensitive and reliable means to align the lens to the $\mathrm{x}$-ray optical axis.

All of the lenses fabricated exhibited focussing properties in the x-ray energy range of $8-16 \mathrm{keV}$. The earlier lenses in particular showed focussing with gain in the higher part of the energy range, still focussing but losing flux appreciably as the energy was reduced below $10 \mathrm{keV}$. Most likely, this was due to the additional PMMA acting as absorber due to the thicker septa walls. The first lenses also showed appreciable aberration and graininess as a variation in the width and intensity of the line from top to bottom of the focus. Typical of the best result measured for these lenses was a focus width of 40 $\mu \mathrm{m}$ FWHM at $12 \mathrm{keV}$ with a gain of 2.5. The newly designed lenses performed appreciably better, showing good focus as low as $8 \mathrm{keV}$. These lenses showed less variation in the quality of the focus from top to bottom and much less graininess. Typical of the best result measured for these lenses was a focus width of $16 \mu \mathrm{m} \mathrm{FWHM} \mathrm{at} 11.5 \mathrm{keV}$ with a gain of 7 . When comparing the planar parabolic lenses to the cylindrical lenses from the same set of three compound lenses, however, there was no significant difference in the quality of the focus. This suggests that the improvements are primarly due to better fabrication, mask quality, and reduced path length through the PMMA, rather than the difference between the two figures. Since the two figures only exhibit appreciable departure from each other in the outer part of the lenses, we conducted a test to determine the effective aperture of the lenses by reducing 


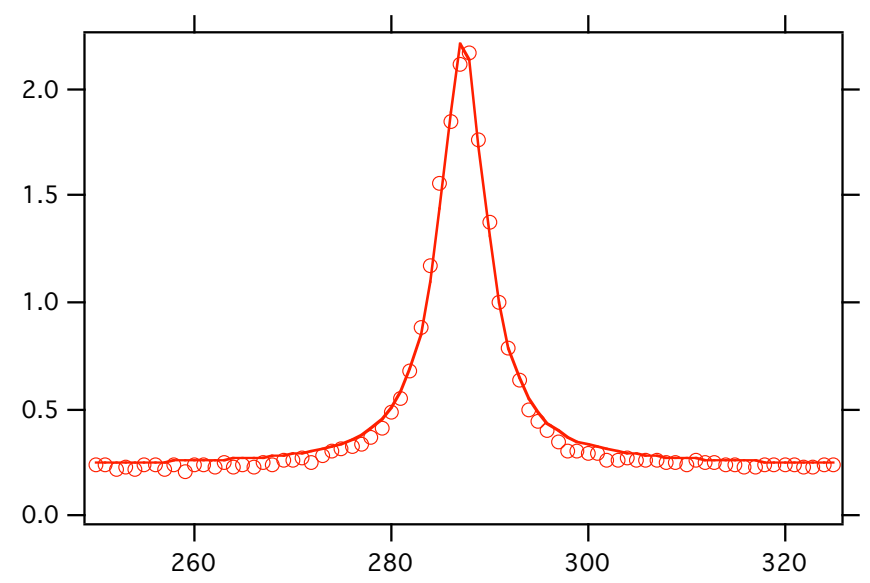

Figure 9. Vertically summed pixel intensities of the line focus of a compound x-ray lens.

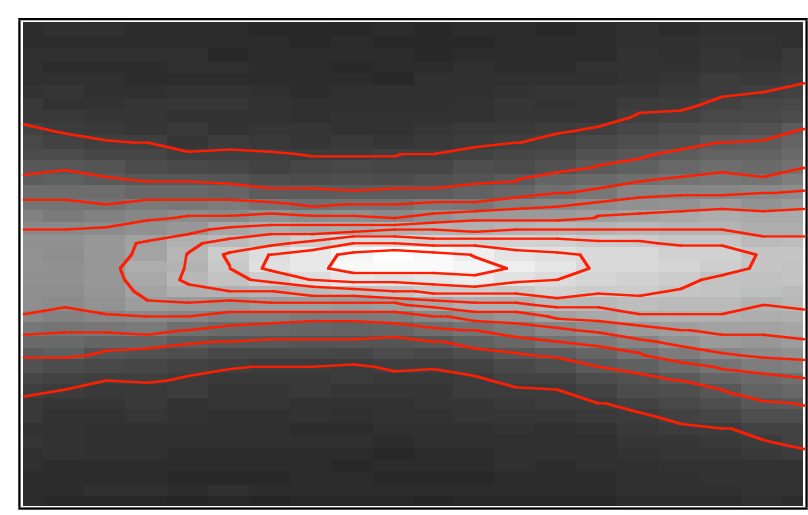

Figure 10. Waist of the focus of a compound $\mathrm{x}$-ray lens obtained from a series of images.

the width of the beam until the intensity in the focus was reduced by $10 \%$. By this method, we determined that only $0.5 \mathrm{~mm}$ width of the lenses contribute to $90 \%$ of the intensity. Moreover, the lenses are likely still limited in quality of focus by the errors in figure that occur near the septa, as observed by optical microscopy. This suggests that significant improvement can be had by improving the method of fabrication. For example, the figure accuracy of the septa may be better maintained if the structures are anchored onto a substrate instead of being fabricated in a freestanding PMMA sheet. Nevertheless, the quality of the newer lenses significantly improved over previous versions these lenses and should be of use to those who would otherwise simply reduce the size of the beam below $20 \mu \mathrm{m}$ with slits.

\section{CONCLUSIONS}

Compound refractive x-ray lenses have been fabricated using DXRL. Early lenses were produced directly in 1- to 6-mm-thick sheets of PMMA with more than 85 cylindrical lenses along the optical axis. These early lenses were improved upon by optimizing the design of the lenses and improving mask quality. The newer compound lenses include 100 planar parabolic biconvex lens elements with features to provide easy separation of sets of compound lenses. The lenses are shown to be suitable for use in synchrotron radiation from bending magnet radiation at the Advanced Photon Source in the energy range of $8-16 \mathrm{keV}$. The lenses can provide line focus with $16 \mu \mathrm{m}$ FWHM and intensity gain of 7 with effective horizontal aperture of $0.5 \mathrm{~mm}$ and vertical aperture of $2.5 \mathrm{~mm}$. Lens performance still remains limited by quality of fabrication, so that further improvement may be possible.

\section{ACKNOWLEDGMENT}

This work was supported by the U.S. Department of Energy, Office of Science, Basic Energy Sciences, under Contract No. W-31-109-Eng-38. 


\section{REFERENCES}

1. H.D. Bauer, W. Ehrfeld, M. Gerner, and T. Paatzsch, "Micro-optical elements and optical interconnection components fabricated using the LIGA technique," Proc. Intl. Symp. Microsys., Intellig. Materials, Robots, Sendai, Japan, 1995.

2. A. Snigirev, V. Kohn, I. Snigireva, and B. Lengeler, "A compound refractive lens for focusing high energy x-rays," Nature 384, 49-51, 1996.

3. B. Cederström, "X-ray focusing using refractive lenses in a digital x-ray imaging system," Master's, Lund Institute of Technology, 1997.

4. R. K. Kupka, M. Roulliay, F. Bouamrane, S. Megtert, "Modern VUV and X-ray micro-optics: LIGAfabricated and micromachined devices," Proc. SPIE 3512, 398-409, 1998.

5. R. K. Kupka, F. Bouamrane, M. Roulliay, S. Megtert, "X-ray transmission lenses by deep x-ray lithography and LIGA technique: First results and fundamental limits," Proc. SPIE 3680, 508-517, 1999.

6. D. C. Mancini, N. A. Moldovan, R. Divan, F. DeCarlo, J. Yaeger, "Fabrication of refractive x-ray lenses by deep x-ray lithography," presented at SPIE Annual Meeting, San Diego, CA, August 2000.

7. N. Moldovan, D. C. Mancini, R. Divan, O. V. Makarova, A. Peele, K. Podolak, "Sidewall roughness in ultra-deep x-ray lithography," submitted to Microsystems Technologies Journal.

8. B. P. Lai, D. C. Mancini, W.-B. Yun, E. S. Gluskin, "Beamline and exposure station for deep x-ray lithography at the Advanced Photon Source," Proc. SPIE 2880, 171-176, 1996.

9. C. H. Malek, S. Yajamanyam, "Evaluation of alternative development process for high-aspect-ratio poly(methylmethacrylate) microstructures in deep x-ray lithography”, J. Vac. Sci. Technol. B 18 (6), 3354, 2000.

10. R. Bruck, K. Hahn and J. Stienecker, "Technology description methods for LIGA processes," $J$. Micromech. Microengin. 5, 196-198, 1995. 